\title{
Teaching Special Relativity: Developing a Software Aid for Spacetime Diagrams
}

\author{
Randy Wolfmeyer \\ Department of Chemistry and Engineering Physics \\ University of Wisconsin-Platteville \\ John Wood Community College ${ }^{1}$
}

\begin{abstract}
Special Relativity is an important subject for the space sciences, but is often difficult for students to understand. Spacetime diagrams provide a graphical tool to aid in the conceptual understanding of relativity. The SpaceTime applet is designed to aid students in drawing spacetime diagrams and setting up diagrams for specific problems. A lab activity is also developed for use with the applet in studying spacetime diagrams.
\end{abstract}

\section{Introduction}

Special relativity is considered to be a difficult subject by many students - it is often not well understood by even advanced graduate students ${ }^{4}$. It involves concepts that we simply do not experience in our day-to-day lives, and contradicts our common sense concepts of a time and space. It was not until the development of Maxwell's equations and the negative results of the Michelson-Morley experiment in 1887 that there was even a hint of this more complex nature of the universe, and it was another 18 years until Einstein was able to make sense of the results. It is quite understandable that students still struggle with understanding this subject. Fortunately, Einstein worked out the theory of special relativity from a simple set of postulates, with the seemingly fantastic results of time dilation and length contraction following as inevitable logical consequences. Traditional textbooks, however, often present special relativity as a set of confusing equations with little emphasis on giving the students a conceptual understanding of the reasoning behind the equations - instead students rely on plug-and-chug techniques to get to an answer that the textbook says is correct. Students have difficulty understanding the results, or simply do not believe the results.

Special relativity is a prerequisite topic of study for many space related sciences, especially cosmology and many fields of astronomy, and even finds its way into precision calculations for spacecraft, not to mention exploration of far-future forms of propulsion, all components of NASA's goals for science missions, especially those related to astrophysics explorations. Many students have an interest in the topic, but the traditional way of teaching it causes many students to become disinterested. Teaching special relativity so that students gain understanding of the concepts and can follow the logical implications can increase student interest in further study of space sciences. Introductory courses in physics that have successfully introduced special relativity have found that students are genuinely excited to learn and understand such a wellknown but seemingly complex subject ${ }^{2}$.

1This work is supported by the Wisconsin Space Grant Consortium through the Higher Education Incentives grant. 
Basic Principles of Special Relativity. Einstein's Theory of Special Relativity has its roots in the development of Maxwell's Equations governing electricity and magnetism. The four equations lead to a wave solution that allows electromagnetic energy to transmit as a wave, with a predicted wave velocity of $\mathrm{c}=3 \times 10^{8} \mathrm{~m} / \mathrm{s}$, the speed of light. But a conundrum presented itself; the electromagnetic waves predicted by Maxwell's Equation made no reference to a physical medium with respect to which one could measure the speed of light. The ether was proposed as the medium for the electromagnetic waves, with Maxwell's Equations being complete in a reference frame at rest with respect to the ether, but requiring additional correcting terms in other frames moving with respect to the ether. However, the Michelson-Morley experiment of 1887 failed to detect the change in the speed of light due to the Earth's motion through the ether.

Einstein proposed a solution with his Theory of Special Relativity in $1905^{1}$. His theory is contained within two postulates: 1. The laws of physics are the same in all inertial (nonaccelerating) references frames, and 2 . The speed of light is measured the same by all observers in inertial reference frames. The first postulate assumes that Maxwell's equations must be valid in all inertial reference frames without frame specific correction terms. The second postulate is essentially a logical conclusion of the first; no matter the relative velocity all observers will observe light moving at the same velocity. This seems absurd. According to Newtonian physics, two observers moving relative to one another will obviously measure different speeds for a third object. For example, what happens if you are traveling $90 \%$ of the speed of light and you turn on your headlights? Einstein's answer is that you will record the light traveling at the same $3 \times 10^{8}$ $\mathrm{m} / \mathrm{s}$ that a stationary observer would record.

The solution to this paradox is that Einstein recognized that time is not universal. Two observers moving relative to one another will not measure the same time intervals between two events. It also leads to the concept of non-simultaneity, that two observers may not agree on whether or not two events occur at the same time.

The Lorentz transformation equations:

$$
t^{\prime}=\gamma\left(t-\frac{v x}{c^{2}}\right) \quad x^{\prime}=\gamma(x-v t) \quad \text { where } \quad \gamma=\frac{1}{\sqrt{\left(1-v^{2} / c^{2}\right)}}
$$

give us the means to mathematically translate the time and spatial coordinates of an event from one frame to another. In these equations, $x$ and $t$ are the position and time of an event in one frame, while $x^{\prime}$ and $t^{\prime}$ correspond to the position and time of the same event as observed from another frame moving at speed $v$ with respect to the first frame. The term is known as the Lorentz factor, appearing in many special relativity equations. These equations provide a quantitative way to calculate the observations made by different observers.

From the Lorentz transformation equations, we can also derive the equations for time dilation (time slows down for objects observed to move at relativistic speeds) and length contraction (the length of objects moving at relativistic speeds are shortened):

$$
\text { Time Dilation: } \quad \Delta T^{\prime}=\gamma \Delta T_{0} \quad \text { Length Contraction: } \quad L=\frac{L_{0}}{\gamma}
$$

where $\Delta T_{0}$ is the proper time between two events, $\Delta T$ is the dilated time, $L_{0}$ is the rest length of an object, and $L$ is the contracted length. 
This is the point at which many introductory textbooks end their discussion of Special Relativity. The equations are not difficult to manipulate mathematically, but students often find the equations confusing. Given a specific problem they have difficulty assigning values to the variables in the equations, or confuse the signs for relative velocities. Once they have an answer, they find it difficult to verify a correct answer because the scenarios in special relativity are outside the realm of our normal experiences and often non-intuitive so that it is difficult to judge if an answer "makes sense". The students have difficulty gaining a conceptual understanding of what the equations physically represent.

Spacetime diagrams. A spacetime diagram is a useful tool that can be used to help students gain a conceptual understanding of special relativity. Used properly, spacetime diagrams provide a graphical illustration of the non-Euclidean geometries involved and a way to solve complex problems without relying solely on quantitative equations. By analogy, a well drawn spacetime diagram serves a similar purpose as the free-body diagram in a complex mechanics problem; it allows one to more easily visualize the geometry and arrive at an intuitive understanding of the solution before crunching numbers.

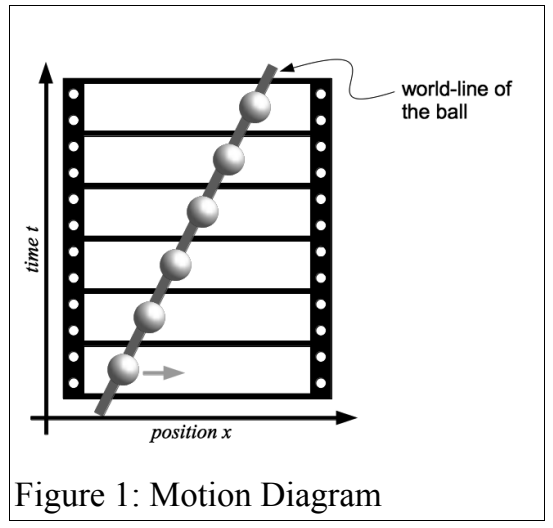

Spacetime diagrams provide a simplified model of relativistic motion in one space dimension, usually oriented along the $\mathrm{x}$-axis, with a set of events that occur at single points in space and single instants of time. A spacetime diagram is essentially a motion diagram, like a filmstrip of a moving ball with each frame an instant of time, and its position in each frame marked by an event. Connecting the events together we construct the "world-line" of the moving ball as shown in Figure 1.

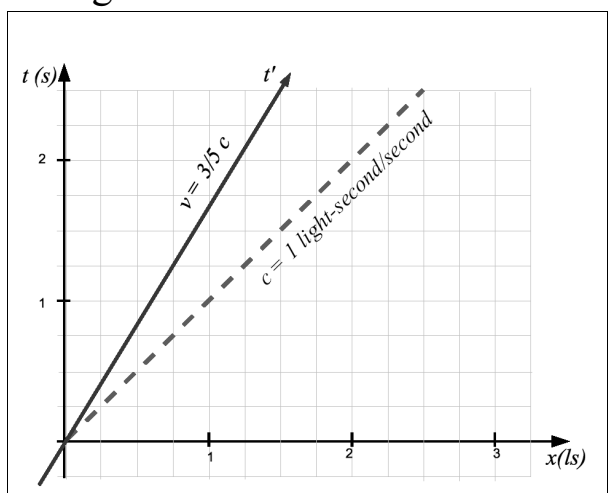

Figure 2: $t^{\prime}$ axis and the speed of light.
Since problems in special relativity involve velocities close to the speed of light, we tend to choose units that simplify the diagrams. A common convention is to measure position in light-seconds (ls), or the distance that light travels in 1 second $\left(1 \mathrm{ls}=3 \times 10^{8} \mathrm{~m}\right)$. This has the advantage that we can illustrate the speed of light on the diagram as a line with a slope of 1 ls/s, as shown in Figure 2.

So far we have considered the motion from one reference frame, but special relativity depends on the observations from different inertial reference frames. To construct a twoobserver spacetime diagram, we can place the origin of a coordinate system for an observer on the moving object. Since this observer assumes that the reference frame is stationary (there are no special reference frames and all inertial motion is relative) we place the spatial origin of their coordinate system on the world-line of the moving object, and label the world-line the $t^{\prime}$ axis. By convention established by Tom Moore ${ }^{2}$, we call the reference frame moving in the $+x$ direction the Other Frame, and the frame moving in the $-x$ direction (with respect to the Other Frame) the Home Frame. The faster the Other Frame moves with respect to the Home Frame the more the slope of $t^{\prime}$ axis rotates toward the slope of the speed of light. 
Due to the Lorentz transformation equations, the scaling of time along the $t^{\prime}$ axis is not the same as on the $t$ axis. One can use the time dilation equation to determine the appropriate scaling along the $\mathrm{t}^{\prime}$ axis. Assume an event occurs at $\mathrm{t}^{\prime}=1 \mathrm{~s}$ along the origin of the Other Frame (along the $t^{\prime}$ axis). Use the time dilation equation to find determine the corresponding time that the event occurs in the Home Frame, then place a tick-mark for $\mathrm{t}^{\prime}=1 \mathrm{~s}$ along the $\mathrm{t}^{\prime}$ axis at the calculated time in the Home Frame. Figure 3 illustrates the procedure for the Other Frame moving at $3 / 5$ the speed of light.

To keep the speed of light constant in both the Home Figure 3: Calibrating the t' axis Frame and the Other Frame(the $2^{\text {nd }}$ Postulate of Special Relativity), the $x^{\prime}$ axis also rotates towards the speed of light line, as shown in Figure 4. The $\mathrm{x}^{\prime}$ axis can be calibrated in a similar way as the $t^{\prime}$ axis, using the length contraction equation. The rotation of the coordinate axes is similar to the transformation for the rotation of the coordinate system about the origin in Euclidean geometry, however the rotation in special relativity is non-Euclidean. In normal Euclidean geometry, the distance between two points is defined by $\Delta d=\sqrt{\Delta x^{2}+\Delta y^{2}}$, and this distance is preserved under transformations between coordinate systems. In space-time, a quantity called the spacetime interval is defined as the time between two events measured by an

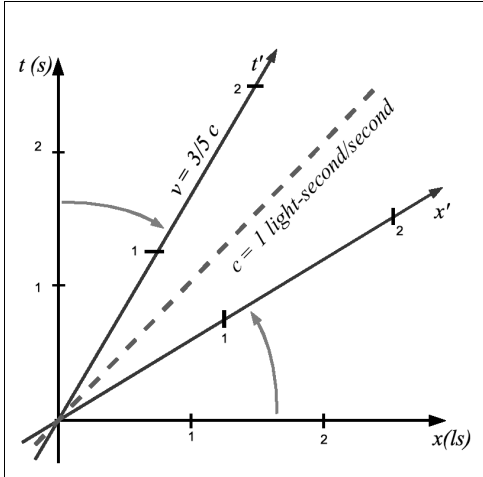

Figure 4: Hyperbolic rotation of the $t^{\prime}$ and $x^{\prime}$ axes

area perpendicular. The process is a bit more complicated as the axes are not perpendicular, leading to coordinate grid that is skewed at an angle. By comparing the coordinate values in each reference frame, an accurately drawn space-time diagram should give the same results as using the Lorentz transformation equations. observer in an inertial reference frame present at both events. The spacetime interval is calculated from spacetime coordinates of two events by an equation similar to that for distance, $\Delta s=\sqrt{\Delta t^{2}-\Delta x^{2}}$. The spacetime interval is preserved under transformations from the Home Frame to the Other Frame and is thus a non-invariant quantity. All observers in inertial reference frames will measure the same value, and this defines many of the geometric properties of spacetime.

By creating a single diagram with two coordinate systems, one can graphically see how the space-time coordinates of an event can be measured in two different reference frames, as shown in Figure 5. Lines are drawn parallel to the axes to connect to the coordinate value on the axes. It is easy to see in the Home Frame, as the axes

The

$$
\text { (1) }
$$

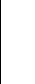
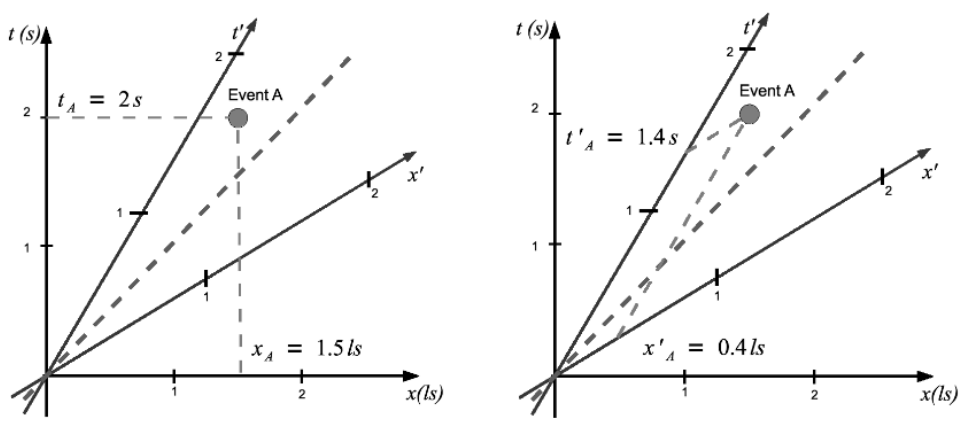

Figure 5: Reading the spacetime coordinates in two reference frames 
The space-time diagram is a very useful tool for graphically establishing problems in special relativity, and can allow students to see conceptual relationships that would otherwise evade them. It can also provide a means for students to evaluate the plausibility of answers obtained from the Lorentz transformation equations.

Experience teaching special relativity with spacetime diagrams has shown that students can interpret a well-drawn space-time diagram, but they have difficulty in setting up their own diagrams to use for a given problem. The students need assistance in the complicated details of setting the coordinate axes, calibrating the axes, and reading the space-time coordinates of an event from a space-time diagram. The goal of this project was to study student understanding in the subject of special relativity, and to develop an interactive software tool that will help students with the creation of spacetime diagrams and illustrate how spacetime diagrams can be used to setup and solve problems in special relativity.

Previous research. While considerable research has been done on how students understand topics in introductory physics courses (forces, motion, electricity, magnetism), substantially less research has been done in the more advanced topics in physics, such as special relativity. There are only a handful of research papers on the subject. Roberto Salgado ${ }^{3}$ has done research on visualizing proper-time in special relativity, and has developed a number of graphical techniques for teaching special relativity. Rachel Scherr ${ }^{4}$ did her dissertation and a series of papers on student understanding of time in special relativity and non-simultaneity. She used a set of questions related to the simultaneity of events in written exams and in-class problems, and conducted one hour interviews to probe student thinking in greater depth. Some of her results were disheartening: "After instruction, $2 / 3$ of physics undergraduates, and $1 / 3$ of graduate students in physics are unable to apply the construct of a reference frame in determining whether or not two events are simultaneous." "It is not surprising that students, even at advanced levels, do not fully understand the implications of the invariance of the speed of light. What is surprising is that most students apparently fail to recognize even the basic issues that are being addressed."

\section{Procedure/Methods}

Student understanding and conceptual difficulties in special relativity. The first step in this project was to study student understanding in special relativity, specifically the use of space-time diagrams, so that tools could be developed to best address student needs. This process involved a variety of techniques, including review of exam results, interviews with students and lab activity/problem solving sessions with students.

Exam results were analyzed from 2 semesters of introductory physics courses one each at Marquette University and John Wood Community College, and 2 semesters of a modern physics course at the University of Wisconsin-Platteville. Each course contained a 2-3 week unit on special relativity, including space-time diagrams with a test after the unit and questions on the

cumulative final exam. Each exam had at least one question requiring the students to setup or interpret a space-time diagram.

Interviews were conducted with a number of student volunteers following one semester of Modern Physics at UW-Platteville. The students were presented problems from special relativity and were asked questions about the problem solving techniques they used and followup questions to determine where there might be conceptual misunderstanding. 
During two courses, one section of Modern Physics at UW-Platteville and one at John Wood Community College, the students used a lab activity developed from earlier interview responses and exam analysis to help students gain practice with drawing space-time diagrams. These results were also studied to add to data on student understanding of special relativity.

SpaceTime applet. To assist students in creating spacetime diagrams and gaining a graphical understanding of the Lorentz transformation equations, the SpaceTime Applet was developed in Java.. This applet was then used by students as part of the lab activity at John Wood Community College.

\section{Results}

Student understanding of special relativity. From the interviews, exam analysis, and problem solving sessions, a number of problem areas were identified within the study of special relativity. The following items were consistently marked on wrong on exams, or areas for which students expressed needing additional help.

Assignment of variables in the Lorentz transformation equations and length contraction and time dilation. Given a problem with a numerical solution, students had difficulty assigning the values from the problem statement to the appropriate reference frame. Especially confusing was the determination of proper time for time dilation problems, and rest length in length contraction problems. The confusion also adds to the conceptual difficulty in the understanding that there are no special reference frames, all inertial reference frames apply the same laws of physics. Proper time and rest length give imply that the values measured within one frame are more "right" than another.

Absolute time and non-simultaneity. Students frequently assume that the results of the Lorentz Transformations are due to light lag - that events appear to be at different times to different observers due to the time for a signal to travel from an event to an observer. They often try to calculate this lag in problems, which can in some situations give the correct numerical answer for the wrong reasons.

Equivalence of inertial reference frames. Historically this was the source of many paradoxes to attempt to disprove special relativity, but it still causes conceptual difficulty for students. Either the students do not apply the principle of relativity and assume special frames, or they misapply the principle to assume all inertial reference frames will see the same results, or they assume the effects negate one another. They fail to make the connection that the apparent paradox is solved by the non-simultaneity of events.

Causal Links. Two events are causally linked if one event can cause another. If a signal between the two events would have to travel faster than the speed of light, they cannot be causally linked and thus can have no influence on one another. Students have difficulty identifying events that can be causally linked as the relationship is determined by both the time and distance between two events.

Each of the classes studied also used space-time diagrams as a graphical tool for studying special relativity, so a number of difficulties with space-time diagrams were also addressed:

Axis orientation. Students do not have difficulty with t' axis, but getting correct angle for the $\mathrm{x}^{\prime}$ axis causes more confusion. Many students fail to understand that the reasoning behind the rotation of the axes is to preserve the invariance of the speed of light. 
Axis calibration. To use a space-time diagram to compare numerical results with the Lorentz transformation equations, the axes must be calibrated. The students could often comprehend the concept of time dilation causing the tic marks on the axis to change, but had difficulty applying the principle to draw their own spacetime diagrams.

Interpreting the space-time diagram. Students often misunderstand the the coordinate grid of the Other Frame is transformed in the same way as the $x^{\prime}$ and $t^{\prime}$ axes, creating a skewed or diamond shaped grid pattern. This mistake usually manifests when students must interpret a space-time diagram. Consequently many students have difficulty determining the sequence of events in the Other Frame.

Space-time interval. The spacetime interval is analogous to distance in Euclidean space, but is hyperbolic being a difference of squares. This makes determining the space-time interval from a space-time diagram an non-intuitive exercise; two events that are farther apart on a diagram can have a smaller space-time interval than two events that are close to each other.

SpaceTime applet. As a result of the study of student difficulty and misconceptions in special relativity and space-time diagrams, the SpaceTime applet was developed to assist the students in drawing space-time diagrams and gaining a graphical understanding of coordinate

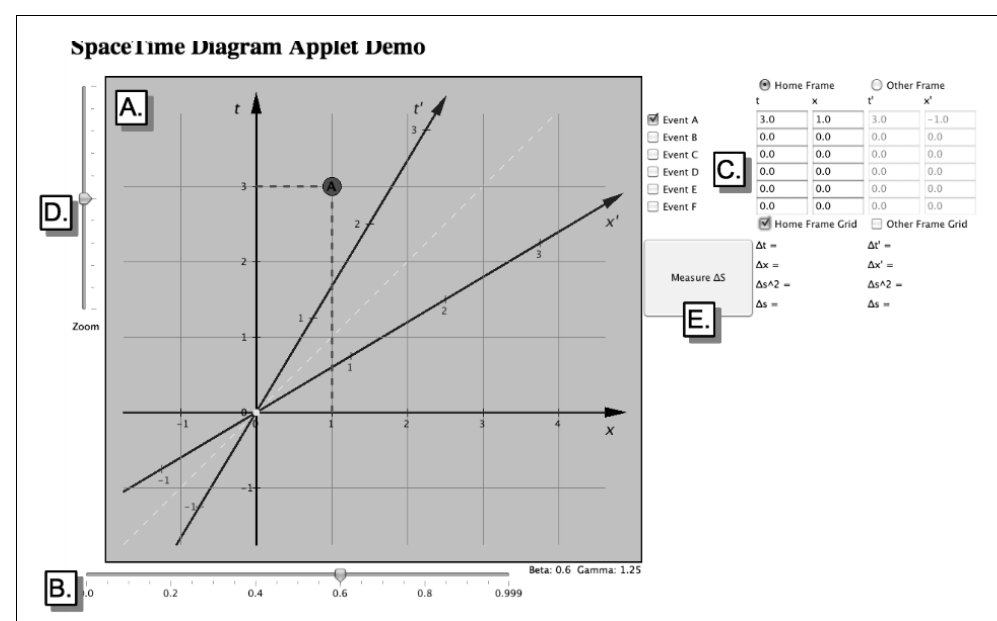

Figure 6: SpaceTime Applet - User Interface transformations and the nonEuclidean geometry of space-time. The SpaceTime applet allows the user to draw a very basic spacetime diagram with up to 5 spacetime events, to determine the coordinates in both the Home Frame and the Other Frame, and to calculate the spacetime interval between two spacetime event.

Figure 6 shows a screenshot of the applet, with the key areas of the user interface indicated.

A) Spacetime Diagram: This is where the two-observer spacetime diagram is drawn by the applet. Elements within the diagram can be selected and moved around by the user.

B) Beta Slider: Slider control to set the value of beta, $(\beta=v / c)$, the relative speed of the Other Frame with respect to the Home Frame. It also calculates the value of gamma, the Lorentz factor.

C) Spacetime Event List: Displays the coordinates of the spacetime events that can be displayed within the diagram, and allows the user to change the coordinates, and set gridline for the display.

D) Zoom Slider: Adjusts the scale of the diagram. Moving the slider upward will zoom into the diagram while moving it downward zooms out allowing the user to adjust the scale to fit a particular problem.

E) Spacetime Interval Calculator: The button activates the SpaceTime Interval measurement tool. 
Uses for the SpaceTime Applet. The SpaceTime Applet addresses a number of issues for students in understanding space-time diagrams.

Correct orientation of the $t^{\prime}$ and $x^{\prime}$ axes: The user can change the relative speed of the Other Frame by adjusting the value of the Beta slider (B) and see how the slope of the $t^{\prime}$ and $x^{\prime}$ axes both tilt toward the diagonal line indicating the speed of light.

Calibration of the scales on the $t^{\prime}$ and $x^{\prime}$ axes: By adjusting the Beta slider (B) and changing the relative speed of the Other Frame the user can see how the scaling on both the $t^{\prime}$ and $\mathrm{x}^{\prime}$ axes are changed. This also provides a basic understanding of time dilation as the user can see the spacing between the $t^{\prime}$ tic-marks increase as the relative speed approaches the speed of light $(\beta=1)$.

Space-time coordinates of an event: The user can add up to 5 events to the space-time diagram (labeled A - F) by clicking on the checkbox next to each event in the SpaceTime Event List (C). The events will default to the origin at $(\mathrm{t}=0, \mathrm{x}=0)$, but they can be altered either by clicking and dragging the event within the space-time diagram drawing area (A), or by entering new coordinates in the Event List (C) and pressing Enter on the keyboard. When the event is selected in the drawing area (A), a pair of dashed lines will connect the event to the coordinate axes to illustrate how the coordinates are determined.

Space-time coordinates in the Other Frame: The space-time coordinates of an event in both the Home Frame and the Other Frame are listed in the SpaceTime Event List (C). By selecting the Other Frame radio button at the top of the list, the user can change the Other Frame coordinates. When the Other Frame option is chosen, and an event selected, the dashed lines will be drawn parallel to the $t^{\prime}$ and $x^{\prime}$ axes to show how the coordinates are determined in the Other Frame.

Space-time Interval: Pressing the "Measure $\Delta \mathrm{s}$ " button (E) activates the space-time interval measuring tool. This activates a tool that can be used within the space-time diagram area (A) to calculate the space-time interval, $\Delta \mathrm{s}$, between two points in space-time. The tool is represented as a dimension line between two circular end points which can be dragged with the mouse to select different points. The end points will "snap" to an event in the diagram. Coordinate lines are drawn to the axes for the currently selected frame in the Event List (C) to indicate the time and space intervals between the two selected points in that reference frame. Choosing a different frame will cause the coordinate lines to switch to the coordinate axes for that frame. In the region next to the "Measure $\Delta \mathrm{s}$ " button(E) the value of the time interval is indicated for each reference frame ( $\Delta t$ and $\Delta t$ ) as well as the value of the space interval

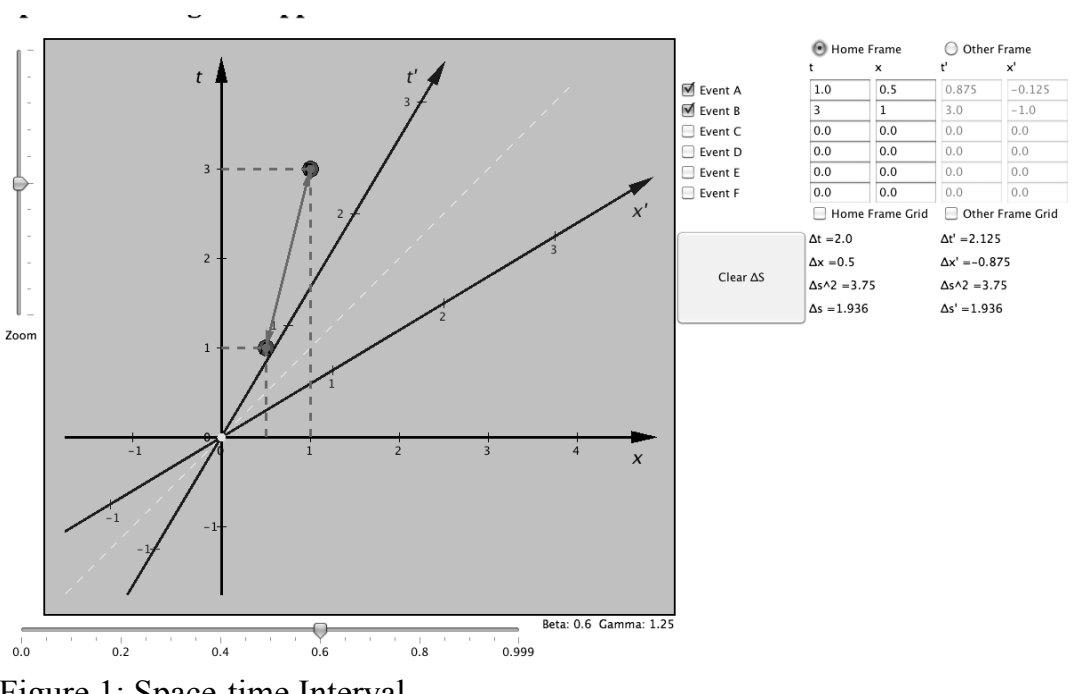

Figure 1: Space-time Interval 
( $\Delta x$ and $\Delta x$ ). The calculation for the space-time interval is shown for each reference frame $(\Delta s$ and $\Delta s$ ) and it should be clear that the two values are always the same no matter the two points chosen in space-time or the relative speed between the two reference frames.

A lab activity was developed utilizing the SpaceTime Applet to introduce students to space-time diagrams and is available online. This activity was designed to be used to either reinforce lecture material on space-time diagrams, or as a standalone activity to illustrate another useful tool in understanding special relativity. All relevant terms are defined in the lab activity and the students are allowed to discover some of the geometrical properties of space-time through their own experimentation. The activity also demonstrates how to use the applet to setup a complex problem in special relativity involving non-simultaneity of events.

\section{Conclusions}

The SpaceTime Applet has been a successful tool for aiding students in understanding space-time diagrams and geometric relationships of special relativity. Students have responded favorably to both the application and the lab activity that guides the students in using the tool to setup spacetime diagrams to solve special relativity problems.

The SpaceTime Applet is a work in progress. Initial testing with both the students and other instructors have indicated a number of bugs that should be fixed, several items that could use improvement, or features that should be added to make the application more useful as a student and teaching aid.

Near future work. Following is a list of items for the next few months.

User Interface. The current user interface is sometimes non-intuitive for users. Additional testing and development with students and instructors must be completed to determine the most effective user interface design. Currently the user can only interact with events and the space-time interval measurement within the space-time diagram itself. Future development would allow the user to adjust the slope of the $t^{\prime}$ or $x^{\prime}$ axes by clicking and dragging the axes. The user should also be able to rescale the diagram and move the origin of the diagram with the mouse.

Event List. Currently the user can only enter 5 events, but it is easy to conceive problems or demonstrations that would require more than 5 events. Ideally the applet would be able to handle any number of events. This requires a different method for adding events and listing coordinates of the events. An idea under development is to add events with a button, and to show the space-time coordinates for an event that is currently selected within the space-time diagram. A complete list of events and space-time coordinates would be provided as a pop-up window after clicking a different button. This should also allow events to be given user selected labels to match the descriptions in different problems or scenarios.

Gridlines. There is currently a glitch in the drawing of gridlines for the Other Frame. Only gridlines for that intersect with the tic marks on the $t^{\prime}$ and $x^{\prime}$ axes are drawn. When the Beta slider approaches $\beta=1$, the coordinate grids by design become very skewed, but the result appears odd because so few gridlines appear on the diagram.

Long term development. The following is a list of items to develop over the next year.

Units. The applet currently sets no specific units, assuming that both the time and space coordinates are using similar units, seconds in time and light-seconds in space for example. 
Students may use textbooks that use specifically defined of units, or wish to keep the SI units. The applet should have an easy option to adjust the units accordingly, and have all calculations done in the chosen units.

World-lines. In addition to events that exist at a single instant of time, many problems in special relativity have objects that persist over time that are better represented by world-lines. Users would have an interface button to add a world-line with a specific speed, and therefore slope, with respect to the Home Frame or the Other Frame. This world-line would be adjustable within the space-time diagram itself, and also indicate intersections with other world-lines or the coordinate axes.

Space-time movies. An advanced feature would allow users to set up events within the space-time diagram, and then view a small "movie" that illustrates the sequence of events as viewed from each reference frame, essentially taking a slice of the diagram for each time step. Events and objects would be represented in the movie by built-in icons for planets, earth, rockets, trains, cars, people, light pulses, etc. These movies would be especially useful for illustrating the concepts of time dilation, length contraction and non-simultaneity of events by isolating the view point from each reference frame.

Teaching mode and demonstration mode. The applet in its current form is very useful for students as a demonstration and an aid for creating space-time diagrams for problems in special relativity. It has been proposed to develop a teaching mode or version that could be used for labs and activities that would allow students to develop their skills in reading information from the diagram and learn some of the mathematical techniques without having the results calculated and displayed by the applet. This mode could be toggled by an instructor to allow the students to use all of the features after completing a set of exercises to demonstrate proficiency.

If you would like to try the SpaceTime Applet, it is available at:

http://www.uwplatt.edu/ evensenh/SpacetimeLab/SpaceTime/SpaceTime.html

The spacetime diagrams lab activity is available at:

http://www.uwplatt.edu/ evensenh/SpacetimeLab/SpacetimeLab.pdf

\section{References}

[1] A Einstein, On the Electrodynamics of Moving Bodies, Annalen der Physik. 17:891, 1905

[2] T. Moore, Six Ideas That Shaped Physics, Unit R: Laws of Physics are Frame-Independent , $2^{\text {nd }}$ Edition, McGraw-Hill

[3] R. Salgado, Physics Teacher (Indian Physical Society), v46, pp. 132-143 (October-December 2004)

[4] R.E. Scherr, P.S. Shaffer, S. Vokos, Physics Education Research, American Journal of Physics Supplement, 69 , S24-35 (2001) 